\title{
Quintupling the speed of the PDP-8 DF32 disk system for real-time use
}

\author{
NOEL, BERNSTEIN \\ Moore School of Electrical Engineering \\ University of Pennsylvania, Philadelphia, Pennsylvania 19104
}

The author has noted some inefficiencies in the operation of the DF32 hardware/software disk system on the PDP-8. While such inefficiencies are not significant when the disk is used only as a store for programs which are called into core from the keyboard, they become important when the disk is used as a real-time backup for core. This paper isolates some of the problems, gives rules for avoiding them, and then describes how the system software was modified to obey these rules. The speed of the modified system is noticeable even when SAVEing and CALLing programs from the keyboard; successive blocks can be transferred between disk and core in one-fifth the previously required time.

\section{HARDWARE}

The DEC DF32 disk ${ }^{1}$ for the PDP-8 is capable of storing 32,768 words. It has a maximum access time (revolution time) of $33 \mathrm{msec}$ and a transfer rate of $66 \mathrm{microsec} /$ word. Each word on the disk is individually addressable, and once core field, starting core address, (negative) word count, and starting disk address have been specified, the disk will read or write the specified number of words $(1-4,096)$ independently of the central processor. At the conclusion of the read or write operation, the word count (which is incremented by one every time a word is transferred) will have been incremented to 0 , and the disk completion flag will come up (and cause an interrupt if interrupts are enabled).

For purposes of this paper, and as far as its actual operation, the disk is best thought of as consisting of 16 tracks of $2,048\left(4000_{8}\right)$ words each, even though computation shows that $(2,048$ words per track $\times .066 \mathrm{msec}$ per word $) /(33$ msec per revolution $)=$ 4 revolutions are required to read or write one track. Apparently the hardware reads or writes only every fourth word during any one revolution.

The reason for considering the disk to be divided into tracks is that experiments by the author have shown that when a track must be crossed during $a$ read or write, one extra revolution ( $33 \mathrm{msec}$ ) is required to perform this track crossing. This only applies to crossing a track during the actual read or write operation. If a read terminates in one track and a new read is initiated in another track, this extra revolution is not incurred.

Thus, our first rule for real-time use of the disk is: Rule 1-Don't cross tracks during individual reads or writes. Nonobservance of this rule can cost up to 15 track crossings (among 16 tracks), or even 15 extra revolutions to read or write the entire disk (which would theoretically take $4 \times 16=64$ revolutions), or $15 / 64=$ $23 \%$ extra time.

\section{SOFTWARE}

For use with most of the DEC-supplied software, the disk is divided into 254 blocks of 129 words each. The blocks are numbered from 0 to $375_{8}$ and can be linked together to form files. Each block contains 128 words of useful information, and the 129 th word contains the number of the next block in the file, or 0 if there are no more blocks in the file.

The method used to allocate blocks to files is straightforward: the first available block is allocated first, then the next available block, etc. This scheme tends to allocate sequential sets of blocks to files, so that the first word of Block $n+1$ of a file is likely to be the word immediately after Word 129 (the link word) of Block n of that same file. When reading Block $n$, the system waits until the link has been read, decodes the link word into the starting address of the next block, and initiates the reading of the next block. However, when Block $n+1$ is physically the next block, by the time the decoding is completed, the first word of the block has already passed the reading heads, and another revolution must be taken before the reading is actually initiated. An unconfirmed experiment by the author has shown that even if the link decoding could be done instantaneously, the disk hardware would still not be able to start reading in time.

Thus we have: Rule 2-Don't let Block $n+1$ of a file start at the word immediately following the last word of Block $n$. Disregarding this rule can cause up to one extra idle revolution for every $1 / 4$ revolution (the space occupied by one block) of reading or writing, or $1 /(1 / 4)=400 \%$ extra time.

\section{SOLUTION}

As has been implied, the DEC-supplied disk system routine violates both of these rules. When using the disk just for saving and calling programs under user control, the 10ths of seconds wasted by this routine are insignificant. However, when using the disk under program control, for example for program overlay, these wasted intervals become significant.

At the suggestion of the author, some users temporarily patched their storage allocation maps 2 to indicate that every other disk block was already in use. Programs they saved then occupied alternate disk blocks, and the extra elapsed time in the Rule 2 case was reduced to $100 \%$. However, using this procedure, unless one is willing to allocate permanently half of the disk to unused filler, deleting and restoring files will partially degrade performance for those files which are restored.

A more complicated but more permanent solution depends on the fact that the programs do not generally perform disk $\mathrm{I} / \mathrm{O}$ operations directly, but usually call on the system I/O routine resident on the last page of Field $O$ to transfer a particular block. Thus, if the block structure is somehow modified to obey our rules, it is only necessary to modify the disk I/O routine's block number decoding algorithm, and all such programs will continue to operate. Unfortunately, PIP and EDIT, two of DEC's supplied programs, and some other programs do perform some of their own disk $1 / O$, and therefore do require further modification, which we have not performed.

In addition to the restrictions implied by our two rules, we are constrained by the fact that we don't want our modified disk $1 / O$ routine to extend over the last page of Field $O$, which is customarily reserved for this routine.

Our solution is based on the following considerations: (1) A maximum of 15129 -word blocks will fit into a 2,048-word track without overlapping tracks. This reduces us to 15 blocks/track $\times 16$ tracks $=$ 240 blocks, numbered 0 to $357_{8}$; or $240 / 254=94 \%$ of total disk space is still available. (2) If we intersperse a few unused words between blocks, we will have time to decode the disk address of the next block before we have passed its first word. (3) If the spaces between blocks are uniform, then the starting address of any. block will remain a linear function of its block number.

A formula which satisfies all of these considerations is: (1) Start Block 0 at Location $10_{8}$ of the disk. 
Table 1

Disk Addresses for the Implemented Routine*
TRACK NO. OO

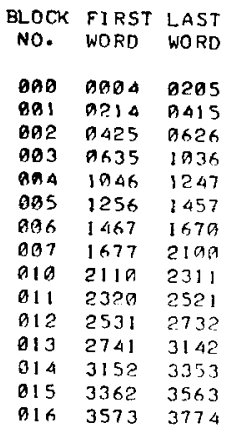

TRACK NO. OI

BLOCK FIRST LAST

NO. WORD WOPD

01700030294

020 9214 5415

021 D424 0625

02205351036

02310451246

$\begin{array}{lll}024 & 1256 & 1457 \\ 025 & 1466 & 1667\end{array}$

$026 \quad 1677 \quad 2100$

92721072319

ด39 23202521

931 2539 2731

$832 \quad 2741 \quad 3142$

83331513352

$\begin{array}{lll}935 & 3572 & 3773\end{array}$
TRACK NO. SA

$\begin{array}{lll}\text { RLOCK } & \text { FIRST } & \text { LAST } \\ \text { NO. } & \text { WORD } & \text { WORD } \\ & & \\ 074 & 9993 & 0204 \\ 075 & 9213 & 0414 \\ 076 & 9424 & 9625 \\ 977 & 9634 & 1935 \\ 190 & 1945 & 1246 \\ 191 & 1255 & 1456 \\ 102 & 1466 & 1667 \\ 103 & 1676 & 2077 \\ 194 & 2197 & 2319 \\ 195 & 2317 & 2526 \\ 196 & 2539 & 9731 \\ 107 & 2749 & 3141 \\ 110 & 7151 & 335 ? \\ 111 & 3361 & 3562 \\ 112 & 357 ? & 3773\end{array}$

TRACK NO. 95

ELOCK FIRST LAST

- WחBT WRD

$\begin{array}{lll}113 & \text { 90म? } \\ 114 & 9213 & 9414 \\ 115 & 0423 & 0624\end{array}$

$\begin{array}{lll}115 & 0423 & 0624 \\ 116 & 0634 & 1035\end{array}$

$\begin{array}{lll}116 & 0634 & 1035 \\ 117 & 1044 & 1245\end{array}$

$120 \quad 1255 \quad 1456$

$121 \quad 1465$ 166h

1221676 2977

$123 \quad 2106 \quad 2397$

$\begin{array}{lll}124 & 2317 & 2520 \\ 125 & 2527 & 2730\end{array}$

$\begin{array}{lll}126 & 2740 & 314\end{array}$

130 3361 3562

(3) 3571 3772
TRACK NO. 11
$127 \quad 3159 \quad 335$
TRACK NO, IB

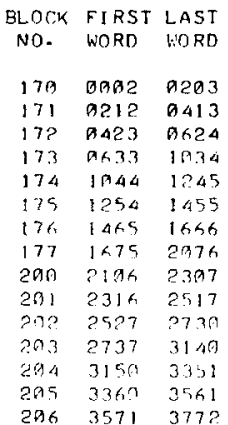

RLOCK FIRST LAST

\section{ND. WOEO SORO}

एबन मดल 929?

?.1० करा? 0413

$\begin{array}{lll}211 & 0422 & 0623 \\ 212 & 0633 & 1034\end{array}$

$213 \quad 1943 \quad 1244$

$214 \quad 1254 \quad 1455$

215 la64 1665

215 1675 2n76

21721052306

$\begin{array}{lll}22.9 & 2316 & 2517\end{array}$

$\begin{array}{lll}221 & 2526 & 2727\end{array}$

$\begin{array}{lll}223 & 3147 & 3350\end{array}$

$\begin{array}{lll}223 & 3147 & 3350 \\ 224 & 3360 & 3561\end{array}$

$\begin{array}{lll}225 & 3570 & 3771\end{array}$
TRACK NO, 14

BLOCK FIRST LAST NO. WORD WORD

2649001 n29? 265 9211 9412

$266 \quad 9422 \quad 0623$

$2670632 \quad 1933$

$279 \quad 1043 \quad 1244$

$271 \quad 1253 \quad 1454$

$\begin{array}{lll}272 & 1464 & 1665 \\ 273 & 1674 & 2975\end{array}$

$2742195 \quad 2396$

$275 \quad 2315 \quad 2516$

$276 \quad 2526 \quad 2727$

$\begin{array}{lll}277 & 2736 & 3137\end{array}$

$330 \quad 3147 \quad 3350$

$302 \quad 3570 \quad 377 \mathrm{I}$

TRACK NO. 15

BLOCK FIRST LAST

NO. WORD VORD

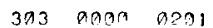

उA न21। 9412

30504210622

$396 \quad 0632 \quad 1933$

$397 \quad 1942 \quad 1243$

$310 \quad 1253 \quad 1454$

$311 \quad 1463 \quad 1644$

$312 \quad 1674 \quad 2975$

3132194 2305

$\begin{array}{lll}314 & 2315 & 2516\end{array}$

$\begin{array}{lll}316 & 2736 & 3137\end{array}$

$\begin{array}{lll}317 & 3146 & 3347\end{array}$

$328 \quad 3357 \quad 3347$

$\begin{array}{lll}329 & 3357 & 3560 \\ 321 & 3567 & 3779\end{array}$

TRACK NO. 62

$\begin{array}{lll}2.3 & 1254 & 1455 \\ .34 & 1464 & 1665\end{array}$

243 3369 356
$301 \quad 3357 \quad 3560$
BLOCK FIRST LAST

NO. WORD WORD

$\begin{array}{lll}036 & 0093 & 0204 \\ 037 & 0214 & 0415\end{array}$

937 म214 0415

$040 \quad 04240625$

04106351036

0421945 1246

$043 \quad 1256 \quad 1457$

$\begin{array}{lll}044 & 1466 & 1667 \\ 045 & 1677 & 2100\end{array}$

$045 \quad 1677 \quad 2190$

047 2329 2521

050 2530 2731

$\begin{array}{lll}951 & 2741 & 3142\end{array}$

$952 \quad 3151 \quad 3352$

$\begin{array}{lll}153 & 3362 & 3563 \\ 054 & 3572 & 3773\end{array}$

TRACK NO. 96

TRACK NO. 12

BLDCK FIRST LAST

BLOCK FIRST LAST

132 DOG2 DPQ3

133 9.13 9414

1340423 0624

135 1634 1935

$136 \quad 1944 \quad 1245$

$137 \quad 1255 \quad 1456$

149 :465 15A6

$142 \quad 21062397$

$113 \quad 2317 \quad 25 ? 9$

$\begin{array}{lll}143 & 2317 & 25 ? 9 \\ 144 & 2527 & 2739\end{array}$

$145 \quad 2749 \quad 314$

$\begin{array}{lll}146 & 3150 & 3351 \\ 147 & 3361 & 356 ?\end{array}$

1503571377 ?

\section{NO. WORD WORD}

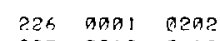

227 ดरा? 0413

$230 \quad 94220623$

23106331934

$\begin{array}{lll}232 & 1943 & 1244 \\ 233 & 1254 & 1455\end{array}$

?.39 $1475 \quad 2976$

$2362105 \quad 2396$

$237 \quad 2316 \quad 2517$

$\begin{array}{lll}249 & 2526 & 2727 \\ 241 & 2737 & 3147\end{array}$

$242 \quad 3147 \quad 3350$

$\begin{array}{lll}244 & 3570 & 3771\end{array}$
TRACK NO. 16

BLOCK FIRST LASI

NO. WORD WORD

322 ดอ8อ ด201

$323 \quad 02110412$

$324 \quad 0421 \quad 0622$

325 9632 1033

$335 \quad 1042 \quad 1243$

$\begin{array}{lll}327 & 1253 & 1454\end{array}$

$3391463 \quad 1664$

$331 \quad 16720075$

$\begin{array}{lll}332 & 2104 & 2305 \\ 333 & 2315 & 2516\end{array}$

$\begin{array}{lll}333 & 2315 & 2516 \\ 334 & 2525 & 2726\end{array}$

$\begin{array}{lll}335 & 2736 & 3137\end{array}$

$\begin{array}{lll}336 & 3146 & 3347\end{array}$

$\begin{array}{lll}337 & 3357 & 3560\end{array}$

$340 \quad 3567 \quad 3770$

TRACK NO. 93

ND. WORO WORD

055 ตอกउ

256 ก213 9414

प.5\% DA34 1035

A6! 19451246

$062 \quad 1255 \quad 1456$

ARS lयAA 1667

6521072310

A66 $2317 \quad 2520$

$967 \quad 2530 \quad 2731$

$\begin{array}{lll}979 & 2536 & 2731 \\ 971 & 3151 & 3141\end{array}$

$0713151 \quad 3352$

97233613562

$973 \quad 3572 \quad 3773$
TRACK NO. QT

BLOCK FIRST LAST

NO. WORD WORD

151 ตดกล ด293

152 9212 9413

$153 \quad 0423 \quad 0624$

$\begin{array}{lll}154 & 0 \times 33 \quad 1934\end{array}$

$\begin{array}{lll}155 & 1344 & 1045 \\ 156 & 1254 & 1455\end{array}$

$\begin{array}{lll}156 & 1254 & 1455 \\ 157 & 1465 & 1665\end{array}$

$1601675 \quad 2976$

$162 \quad 2316 \quad 2517$

$\begin{array}{lll}162 & 2316 & 2517 \\ 153 & 2527 & 2739\end{array}$

$\begin{array}{lll}163 & 2527 & 2739 \\ 164 & 2737 & 3149\end{array}$

$1 \times 5 \quad 3159 \quad 3351$

$\begin{array}{lll}166 & 3369 & 3561\end{array}$
342 a21A MA1I
PLOCK FIRST LAST

957 9424 ब.625

161211962397
TRATL NT. 13

BLOCK FIRST LAST
NO. WDRT WORD

245 क001 0202

$\begin{array}{lll}245 & 6091 & 0202\end{array}$

247 947? 9623

$\begin{array}{lll}259 & 0632 & 1933 \\ 251 & 1943 & 1244\end{array}$

314641665

$\begin{array}{lll}34 & 1674 & 2075 \\ 55 & 195 & 2396\end{array}$

$\begin{array}{lll}255 & 195 & 2396 \\ 256 & 2315 & 2516\end{array}$

$\begin{array}{rll}256 & 2315 & 2516 \\ 257 & 2526 & 2727\end{array}$

$260 \quad 2736 \quad 3137$

$251 \quad 3147 \quad 3350$

$262 \quad 3357 \quad 3560$

$263 \quad 3579 \quad 3771$
$252 \quad 1253 \quad 1454$
TRACK NO. 17

ज. WORD WORD

341 ด9ดด ด2ด1

$343 \quad 0421 \quad 0622$

$\begin{array}{lll}344 & 9631 & 1032 \\ 345 & 1042 & 1243\end{array}$

$346 \quad 1252 \quad 1453$

$\begin{array}{lll}347 & 1463 & 1664\end{array}$

$351 \quad 21042305$

$\begin{array}{lll}351 & 2104 & 2305 \\ 352 & 2314 & 2515\end{array}$

$353 \quad 2525 \quad 2726$

$3542735 \quad 3136$

$\begin{array}{lll}355 & 3146 & 3347\end{array}$

$356 \quad 3356 \quad 3557$

$\begin{array}{lll}357 & 3567 \quad 3770\end{array}$
BLOCK FIRST LAST

*This lable gives the address of the first and last word of each disk block relative to the start of the track in which it falls. It can be seen by inspection that each block lies completely within one track, and Rule $I$ is, therefore, satisfied. It can also be seen that there is a space of at least seven words between each pair of blocks, ana Rule 2 is, therefore, also satisfied. The absolute address of a disk block can be obtained by adding the relative address given here to $4000_{8}$ times the track number. (Note: To allow standard bootstrapping techniques to also work with this system, Block $O$ is moved so that it actually starts at Word $O$ of the disk.)

(2) Leave $7 \frac{1}{2}$ words between blocks ( 7 one block, 8 the next).

The decoding algorithm for converting a block number into the starting address of the block on disk is then: Starting address $=210.4_{8} \times$ block number $+10_{8}$

By exhaustive evaluation (such an evaluation for the algorithm actually used is given in Table 1), it can be seen that all blocks numbered 0 through $357_{8}$ will fall completely within the track they start in. The overhead time required to pass the interblock space when reading or writing consecutive blocks is only $\left(7^{1 / 2}\right.$ words per interblock space)/(129 words per block $)=6 \%$ of minimum time per block, a considerable improvement from the frequently occurring situations previously described.

\section{ACTUAL IMPLEMENTATION}

A slightly modified version of this scheme has actually been implemented by and is in use by the author. This version has the additional feature that the program calling it can be in any field. This is not allowed by the standard I/O routine. With this implementation, the caller's field is indicated by the setting of the data field register. Another special feature is an optional overlapped return in which the routine does not wait for the completion of the $I / O$ operation, but merely initiates it and then immediately returns (through the error return) to the caller.

The calling sequence for the modified DISKIO routine is similar to that for the standard routine. It is called by setting the data field to the caller's field and executing an effective JMS to Location 7642 of Field $O$, followed by six words: Word 1-Bit 1 $=1$ for overlapped return to error return, $=0$ for normal return; Bit $2=1$ for indirect return to contents of link word minus $1,=0$ for normal return; Bits 6-8 = core memory field for 


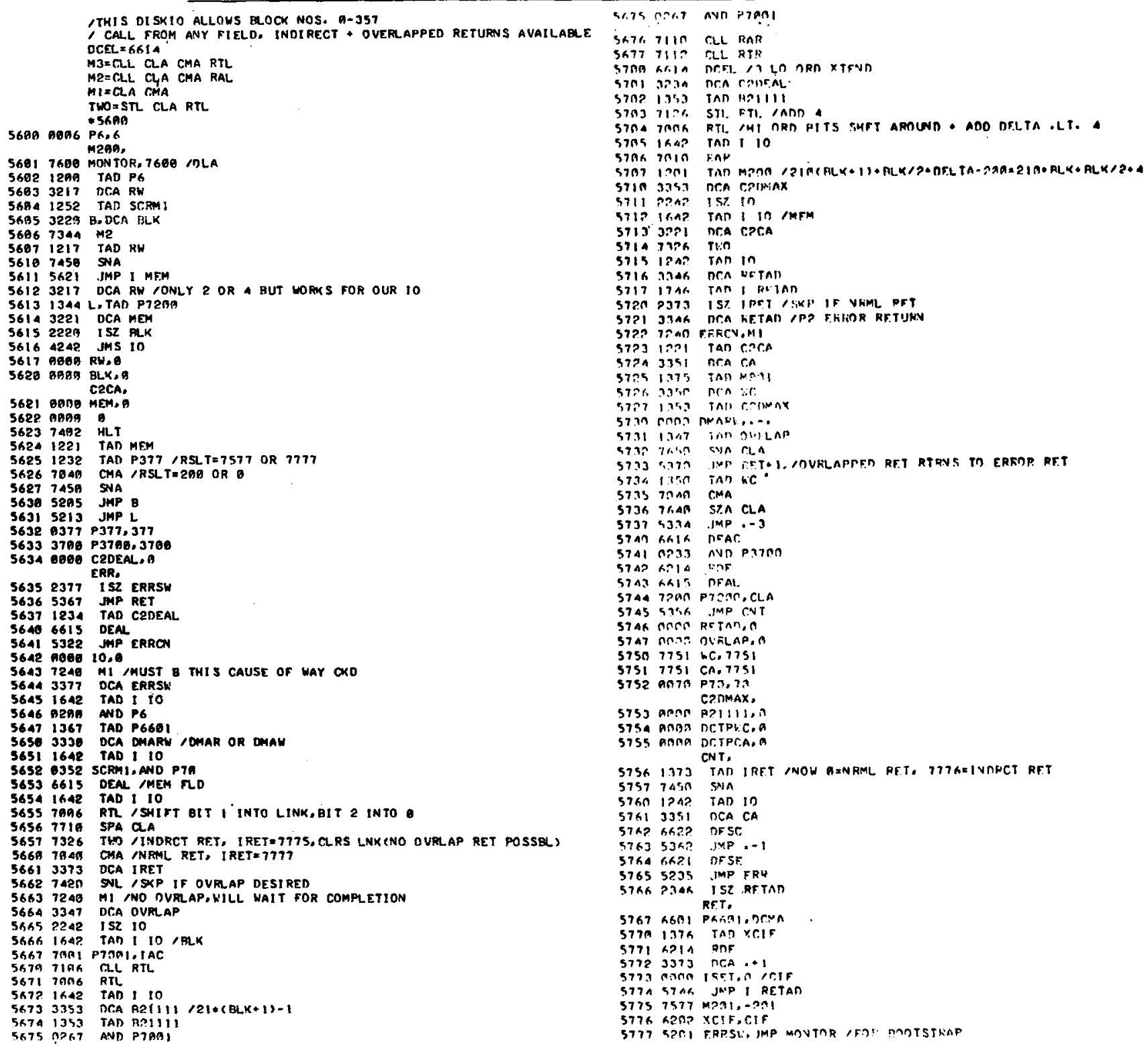

numbers to track addresses performed by the implemented algorithm is given in Table 1 , from which it can be seen that our rules are satisfied.

Since Blocks $373_{8}, 374_{s}$, and $375_{8}$, which are normally system scratch blocks, are nonexistent in our system, program references to these blocks had to be changed. And, naturally, the storage allocation map had to be changed to indicate the additional nonexistent blocks.

The difference in speed when using the modified routine is detectable even when using the SAVE and CALL programs from the keyboard, since the noticeable pause which usually occurs between the entering of a SAVE or CALL instruction and the completion of its execution is now absent. Reads or writes of consecutive blocks under the new system can be done in about one-fifth the time required under the old system.

\section{AVAILABILITY}

The author regrets that only the SAVE, CALL, and DISKIO (SYSIO) routines have been modified to take advantage of these results; other routines which perform their own block-oriented disk I/O will not work properly under this system.

If there is sufficient. demand, a DECtape SWAP $^{5}$ image of a basic modified disk system could be made available to the DECUS library.

\section{NOTES}

1. This paper deals only with the DF32 disk. Because of the hardware restrictions on changing the core field addressed by the disk during a disk read or write, the user must restrict himself either to using only Disk $O$ or to reading and writing only from the core field which contains the disk 1/0 routine, in order to use effectively the results of this paper. Since the author had a configuration which contained only one disk, the former was the course chosen.

2. With standard Moore School operating procedure, each user has his own DECtape SWAP3 image of the dist, and can therefore modify it as he pleases, without adversely affecting other users.

3. Kristol, D. M. SWAP, DECUS. Program 8-154, 1968

4. PDP-8 Disc System Builder Listing, Document No. DEC-08-SBAB-LA, 1967.

5. PDP.8/I Disk Manitor System Document No. DEC-D8-SDAA-D, 1968. 